\title{
Investigating Optimizing Mirror Orbits for Dark-side Illumination
}

\author{
Kaisa E. Crawford-Taylor ${ }^{1}$, Shauna M. Sallmen ${ }^{1}$, Eric J. Korpela ${ }^{2}$ \\ ${ }^{1}$ Physics Dept., University of Wisconsin - LaCrosse \\ ${ }^{2}$ Space Sciences Lab at Univ. of California - Berkeley
}

\begin{abstract}
When a planet is tidally locked with its star, the same side always faces the star; thus one side is always dark. This synchronization occurs quickly for potentially habitable Earth-like planets orbiting dim, low-mass stars. Korpela, Sallmen, \& Leystra Greene (2015; KSG) suggest that advanced extraterrestrial civilizations may put large-scale mirror fleets in orbit around such exoplanets to reflect starlight to the dark side of the planet. They might also use such mirrors to alter the climate of their own or another planet. Radiation pressure (RP) will be important for such large, lightweight mirrors, but research on satellite orbit stability typically neglects its effects. The long-term goal of this research is to determine fuel-efficient satellite orbits in situations where RP is important. We use Python and REBOUND to simulate mirrors orbiting an Earth-like exoplanet in the habitable zone for a variety of stars. Our simulations use two settings: "Always RP" always reflects starlight towards the planet's center while "Nighttime RP" only does so on its dark side. We found mirrors survive longer when initially orbiting face on to the star compared to edge on. We present a selection of results illustrating how RP affects the mirror's survival time.
\end{abstract}

\section{Background and Context}

Finding other habitable worlds has been on the mind of humanity for decades, if not longer. It has been agreed that to harbor life, a planet's surface must be able to hold liquid water. This definition does not include the potential for water-independent life, or life in subsurface oceans. However, typically calculations of habitability include the potential of liquid surface water. Around every star there is a region known as the habitable zone (HZ) where the surface temperature would allow for liquid water. The distance of the HZ from a main-sequence star varies among OBAFGKM star types. The hotter and more luminous the star, the further away the $\mathrm{HZ}$ is. O-type main sequence stars are the most massive, hottest, and most luminous whereas M-types are the least massive, coolest, and dimmest (Bennett et al. 2017). The exact boundaries of habitable zones have been debated as factors such as planet atmosphere, flare activity, and stellar lifetimes also affect water state (e.g. discussion in Petigura et al. 2013).

In an effort to locate habitable planets, the Kepler mission pinpointed 1295 exoplanets of Earthlike radii (from 1.25 to 2 Earth radii) ${ }^{1}$ and 138 of Earth-like masses (from 1 to 10 Earth masses) ${ }^{2}$. These discovered renewed scientific interest in searching for extraterrestrial intelligence.

Korpela, Sallmen, and Green (2015; hereafter KSG) were particularly interested in prospects for detecting the presence of extraterrestrial technologies orbiting Earth sized exoplanets using transit light curves. They modeled Earth-sized exoplanets orbiting in the habitable zone of low-mass stars, which would lead to a higher probability of tidal locking. Tidal locking is a phenomenon where a

\footnotetext{
${ }^{1}$ Retrieved June 18, 2018, from https://exoplanetarchive.ipac.caltech.edu/docs/counts detail.html

${ }^{2}$ Retrieved June 18, 2018, from https://exoplanetarchive.ipac.caltech.edu
}

Funding for this project was provided by the Wisconsin Space Grant Consortium. Faculty adviser is Dr. Shauna Sallmen of the University of Wisconsin - La Crosse. 
planet's day lasts its year, causing a perpetual dark side. Large, lightweight mirrors were hypothesized by KSG to be a possible solution to illuminating the dark side of tidally locked planets or to shade the day side of a planet. These mirrors would have to be large $\left(1 \mathrm{~km}^{2}\right)$ and lightweight $(1000 \mathrm{~kg})$ to be feasible. KSG focused on the effects of a fleet of mirrors on a transit light curve, anticipating them to be more technologically feasible than a single enormous mirror, such as that analyzed by Gaidos (2017). However, this fleet of mirrors assumed an annulus of uniform transparency and did not explore the detailed requirements for specific orbits of such mirrors. Additionally, the research did not include radiation pressure (RP) when analyzing the feasibility of these mirrors.

This project built upon the work of KSG. The long term goal is to model mirror motions and identify fuel-efficient, stable mirror orbits. Using the following equation, acceleration due to RP was calculated to determine its significance in comparison to gravity of the star and planet in the stability of mirrors. In this equation, $\mathbf{L}$ is stellar luminosity (Watts), $\mathbf{s}$ is mirror area (meters ${ }^{2}$ ), $\boldsymbol{\theta}$ is angle of incidence of RP, $\mathbf{c}$ is the speed of light (meters/second), $\mathbf{m}$ is mirror mass (kilograms), $\mathbf{h}$ is planet location relative to the star (meters).

$$
a_{\mathrm{RP}}=\frac{2 \times L \times s}{4 \pi c \times h} \times \frac{\cos (\theta)^{2}}{m}
$$

Fig. 1a shows the ratios of the value for the mirror's maximum acceleration by RP $\left(a_{\mathrm{RP}}\right)$ divided by acceleration due to gravity of the star $\left(a_{\text {Star }}\right)$ and Fig. 1b shows the the equivalent ratio for the gravitational acceleration of the planet ( $\left.a_{\text {Planet }}\right)$. Once the ratio is above $1, a_{\mathrm{RP}}>a_{\text {Gravity. These }}$ situations are for 3 different circular mirror orbit radii for 6 star types with an Earth sized and mass planet in each system. The more massive stars had higher ratios for $a_{\mathrm{RP}} / a_{\mathrm{Star}}$. The larger mirror orbits (higher $\mathrm{R}_{\mathrm{m}}$ ) had higher ratios for $a_{\mathrm{RP}} / a_{\text {Planet. }}$ From these calculations, RP was expected to strongly affect mirror orbit stability in at least some situations.

Acceleration From RP/Gravitational Acceleration from Star

for different mirror orbits $(\mathrm{Rm}=$ Earth Radii)

- $\mathrm{Rm} 2=\mathrm{Rm} 3 \approx \mathrm{Rm} 10$

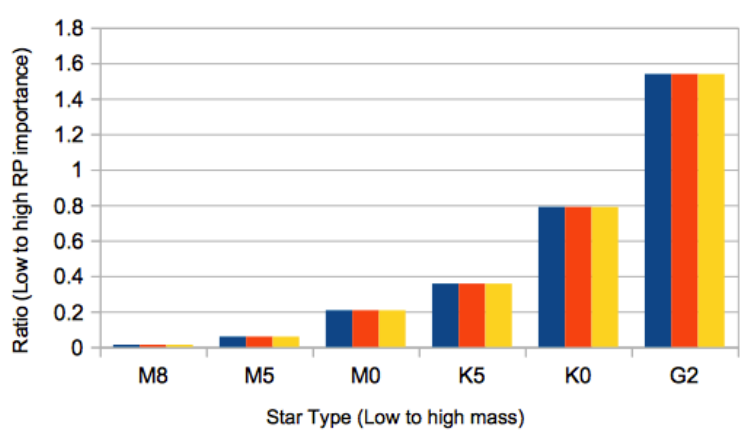

Acceleration From RP/Gravitational Acceleration from Planet for different mirror orbits (Rm = Earth Radii)

- $\mathrm{Rm} 2=\mathrm{Rm} 3=\mathrm{Rm} 10$

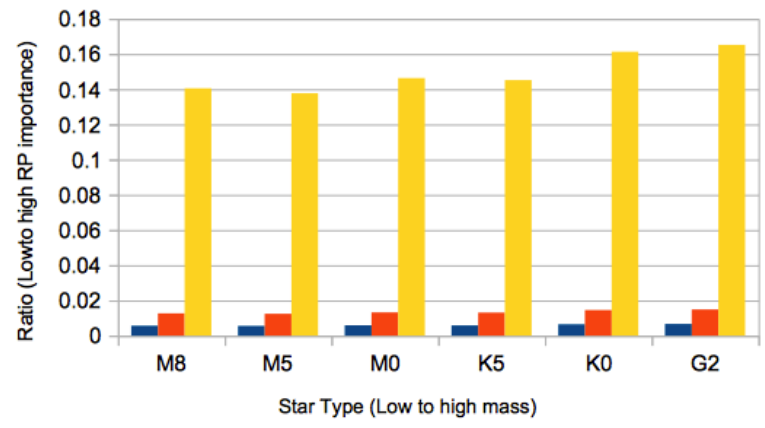

Fig 1. Graphs of $a_{\mathrm{RP}} / a_{\mathrm{Star}}$ (Fig 1a, left) and $a_{\mathrm{RP}} / a_{\text {Planet }}$ (Fig $1 \mathrm{~b}$, right) for various mirror orbit radii $\left(R_{m}\right)$. More massive stars have higher ratios. Larger $R_{m}$ have higher ratios. Larger ratios indicate increased RP significance in mirror stability. 
Correcting destabilized orbits would require fuel-consuming thrusters, which could be resource draining. Hypothetically, one would want to use the least amount of fuel possible, thus one would want to find optimal mirror orbits where RP has a minimal effect on stability. Once ideal orbits are found, the opacity of a mirror fleet and distribution of mirrors can be hypothesized. To our knowledge, no one has simulated accurate orbits where RP was significant. Thus, this project began to extend the work of KSG (2015).

This project does not incorporate stellar wind pressure. The mirrors must be extremely thin to be sufficiently large and lightweight. Therefore high-energy stellar wind particles may well pass through the extremely thin mirrors instead of exerting a force as they are reflected or absorbed (personal communication, Korpela).

\section{Procedures and Methods}

This project studies the motions of large, lightweight, orbiting mirrors in the presence of radiation pressure. We wrote a software package to run these computer simulations. The package is written in Python and utilizes the REBOUND package (Rein \& Liu 2012). REBOUND is an N-body simulator which integrates the motions of $\mathrm{N}$ amount of particles under the influence of gravity. One can also add custom forces such as Stark forces, or in our case, radiation pressure. For testing purpose we have sometimes used REBOUND's WHFast integrator (Rein and Tamayo 2015). For the actual simulations, we use the more accurate adaptive, high-order integrator IAS 15 (Rein and Spiegel 2015). The package was extensively tested for situations with no RP before radiation pressure was added.

We used our software to simulate a $1000-\mathrm{kg}, 1 \mathrm{~km}^{2}$ mirror orbiting an Earth-sized, Earth-mass, exoplanet. The planet orbits a low-mass M, K, or G star at the inner edge of the habitable zone. The initial mirror orbits were circular with an orbital radius $\left(\mathrm{R}_{\mathrm{m}}\right)$ of either 2, 3, or 10 Earth radii. The goal was to figure out which parameters would result in relatively stable mirror orbits under the influence of RP.

In addition to no RP, this project included two modes of RP: "Always" and "Night". Always RP assumed the mirror always redirected starlight to the planet's center. This alignment would raise global temperatures as it also illuminates the day side. For Night RP, the mirror only redirects starlight when on the night side of the planet. This means that Night RP mirrors experience no RP when on the day side, because they are edge-on to the star. This project also used crash and escape detection to end the simulation before the specified number of mirror orbits is reached. If the mirror crashed into the planet or the mirror reached escape velocity, the simulation stopped.

Initial mirror orbits were either coplanar or perpendicular to the planet's orbit. The planet's orbit is in in the XY plane, so the mirror initially orbits in either the XY, XZ, or ZY plane. The package recorded data for 200 mirror orbits or until the mirror reached escape velocity or crashed.

From our runs, we have 162 unique situations simulated. Each simulation outputs tables of coordinate, velocity, and acceleration data over time for the mirror, planet, and star. A simulation also outputs 3 types of graphs. There is an overview plot, which shows mirror and planet positions over time relative to the star. Starlight always comes from the direction of the star. There is a planet-centered graph, which shows mirror positions over time relative to the planet. In this graph, 
the direction of starlight is constantly changing during the simulation. There is also a stationary rotating reference frame (Stationary RRF) graph where the mirror has been transformed to a rotating reference from so that the planet and star appear to "stay still", while the mirror orbits the planet. This has the advantage of keeping the direction of RP constant in the graph as the mirror orbits, but introduces artifacts in the mirror position and motion. Each plot type outputs a graph in the XY, XZ, and ZY planes. To summarize, every run outputs several .csv data tables and 9 graphs of the mirror motion. Each simulation is categorized based on survival time and end state of the mirror - whether it crashed, escaped, or survived until the end of the simulation. Survival time is quoted in number of "mirror orbits", which is calculated using the survival time in units of initial mirror orbit period. Most simulations requested 200 orbits, but if the mirror survived, we ran a new simulation for up to 1000 mirror orbits.

\section{Results and Findings}

Mirrors which orbited at a consistent distance from the planet without the effects of radiation pressure often became unstable once RP was introduced. Fig. 2 shows the Stationary RRF graphs for two situations that are identical apart from the presence of radiation pressure. When looking at the following graphs, what's important is the pattern of the lines, which corresponds to mirror movement. In all graphs of the mirror position, the early data points are colored purple, gradually changing to yellow during the simulation.
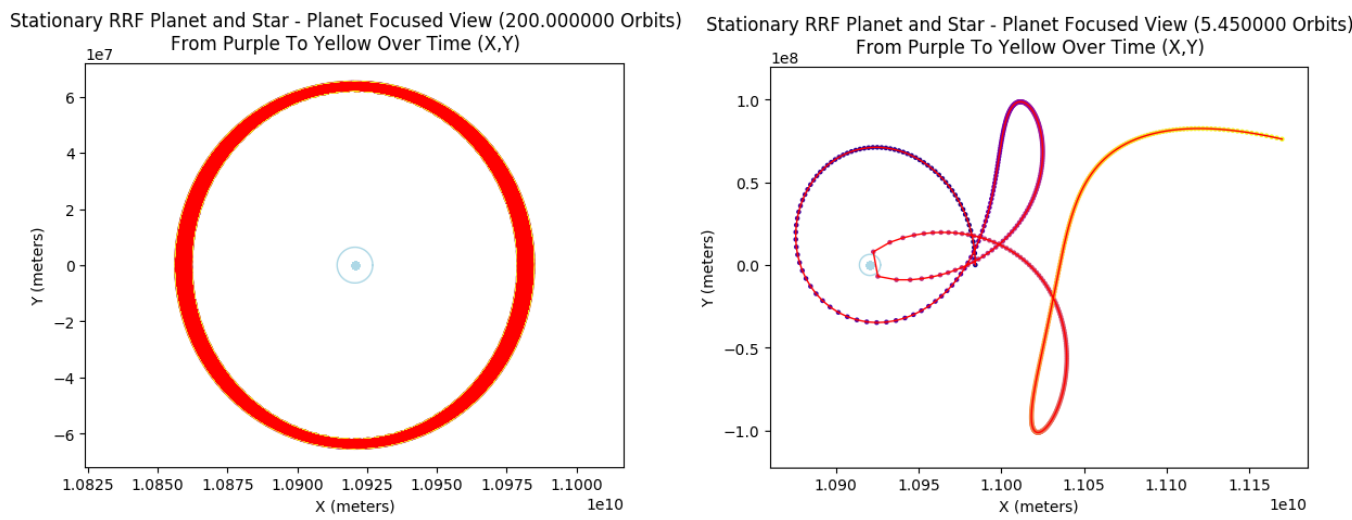

Fig. 2. These are Stationary RRF graphs of the mirror motions for an Earth at the inner edge of the $\mathrm{HZ}$ of an M5 star. Stationary RRF always has RP directed outward from $(x, y)=(0,0)$, i.e. towards the right in these diagrams. The left situation has no RP while the right has Always RP. The left situation appears very stable and the mirror survives the full 200 orbits requested. However, in the right situation the mirror escapes its orbit at 5.45 mirror orbits.

Out of 54 no RP situations, 46 mirrors survived. Those which did not survive (due to gravitational effects of the star) did not survive with RP either. For Always RP, only 12 mirrors survived, and for Night RP, only 10 simulations lasted 200 orbits.

Interestingly, there were two scenarios where RP helped a mirror survive longer. Fig. 3 shows one of these situations using Stationary RRF graphs. A mirror orbiting at $R_{m}=10$ Earth radii in the 
$\mathrm{XZ}$ plane (edge-on orbit) around a Earth at the inner HZ of an M8 star with no RP lasts 0.98 mirror orbits, but with Always RP it lasts 1.18 mirror orbits, and for Night RP it lasts 1.16 mirror orbits. Additionally (not shown), a mirror orbiting at $\mathrm{R}_{\mathrm{m}}=10$ Earth radii around a Earth in the $\mathrm{ZY}$ plane (face-on orbit) at the inner HZ of an M8 star with no RP lasts 1.88 mirror orbits, but lasts 2.94 mirror orbits with Night RP.

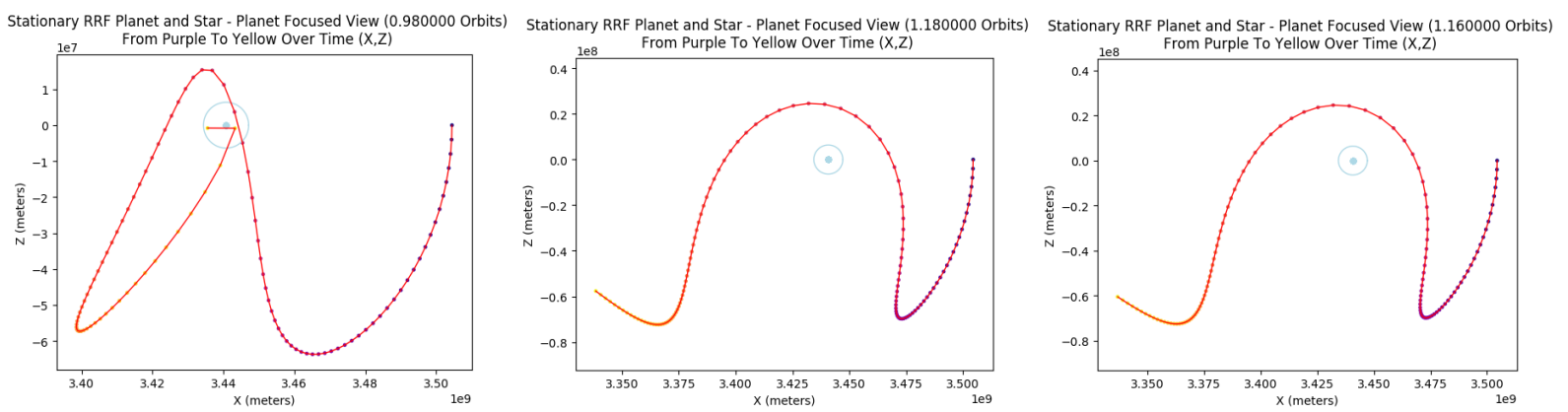

Fig. 3. Stationary RRF graphs of a mirror with $R_{m}=10$ Earth Radii orbiting in the $X Z$ plane around an Earth that is at the inner edge of the HZ of an M8 star. RP Mode (left to right): No RP, Always RP, Night RP.

Overall, initially face-on orbits (see Fig. 4) tended to last longer when compared to initially edgeon orbits. For the standard configuration (where the star, planet, and mirror all begin on the $\mathrm{x}$ axis), a face-on mirror orbit lies in the ZY plane. However, when the simulation begins with the planet on the $+\mathrm{y}$ axis, the face-on orbit is the $\mathrm{XZ}$ plane. Both had nearly identical orbit patterns and survival times.
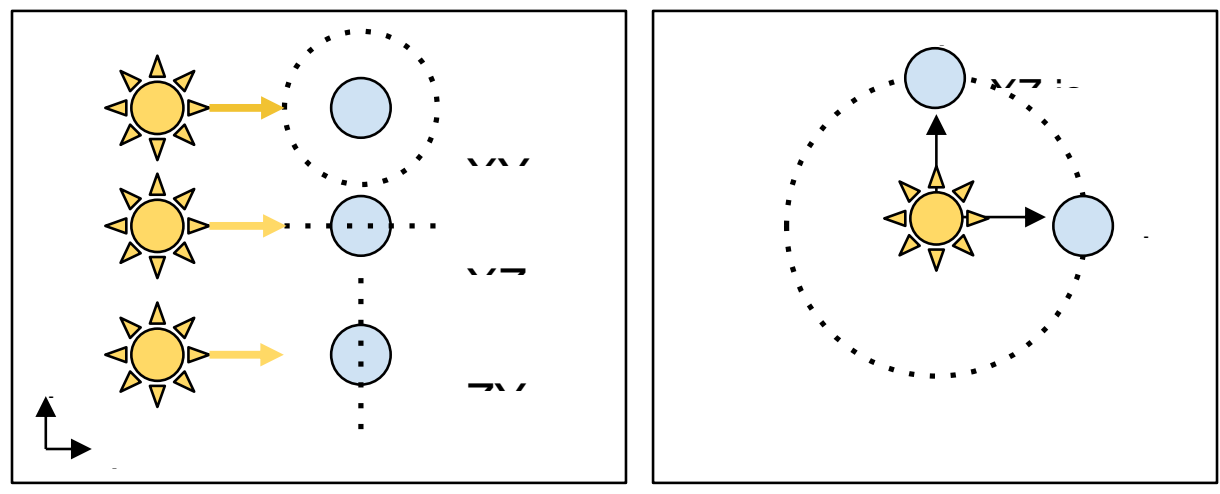

Fig. 4. Schematic of mirror configurations (left). Initially face on orbits tended to last longer than initially edge on. Initial planet location determines which orbit plane is face on or edge on (right).

Fig. 5 shows planet-centered plots in the XY plane for situations that differ only inthe initial mirror orbit plane. The top left initially orbits in the XY plane (edge-on), the top right in the XZ plane (edge-on), and the bottom in the ZY plane (face-on). The initially face-on orbit lasts for the full 200 requested mirror orbits while the XY only survives 11.08 and the XZ only survives 16.99 mirror orbits. Note, the planet is transparent to make the whole mirror orbit path visible, thus if the mirror appears to intersect the planet, it is simply going behind/in front of the planet. 

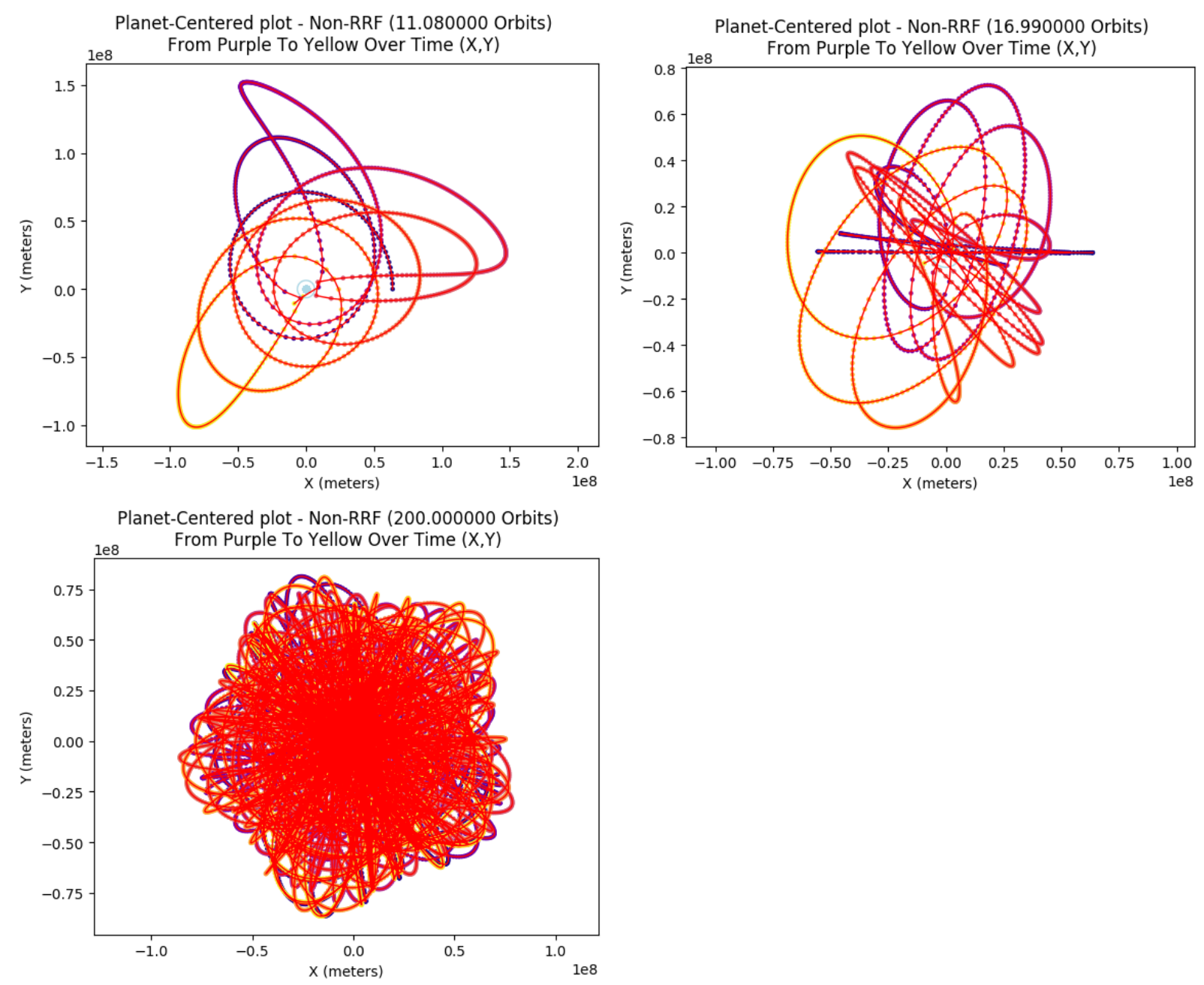

Fig. 5. These $3 \mathrm{XY}$ plane planet-centered graphs are all for the same situation - a mirror orbiting at $\mathrm{R}_{\mathrm{m}}=10$ Earth radii around a Earth at the inner HZ of an M0 star, with Always RP. The top left plot initially orbits in the XY plane, while the top right is initially in the $\mathrm{XZ}$ plane. Both are initially edge-on orbits. The bottom situation is initially face-on in the ZY plane. Note that RP is constantly changing direction in planet centered plots.

The reason face-on orbits lasted longer on average is because there is the least variation in the magnitude and direction of the RP on the mirror throughout a mirror orbit. In these cases, the direction of starlight is nearly 90 degrees to the initial mirror orbit, the mirror is always at a significant angle to redirect the starlight, and the mirror is always about the same distance from the star as it orbits the planet. This trend persisted when testing face-on, $\mathrm{XZ}$ orbits where the planet starts a quarter of the way through its orbit. However, eventually face-on orbits would become edge-on as the planet goes around its star, even if the mirrors were completely stable otherwise.

Fig. 6 shows that for initially face-on mirror orbits with Night RP, radiation pressure often causes the orbit to elongate. The orbital axis then gradually changes direction, i.e. the mirror orbit precesses. A mirror's orbit elongates more quickly for more massive and powerful stars. This is at least partly because for those stars, the HZ lies further out, so there are more mirror orbits per planet orbit. This means the direction of RP relative to the axis of the elongated mirror orbit is more consistent for higher-mass stars. For lower-mass stars, the direction of RP changes in fewer 
mirror orbits, so the elongation effects are less dramatic, and can even largely cancel out. This elongation does not systematically occur for Always RP.
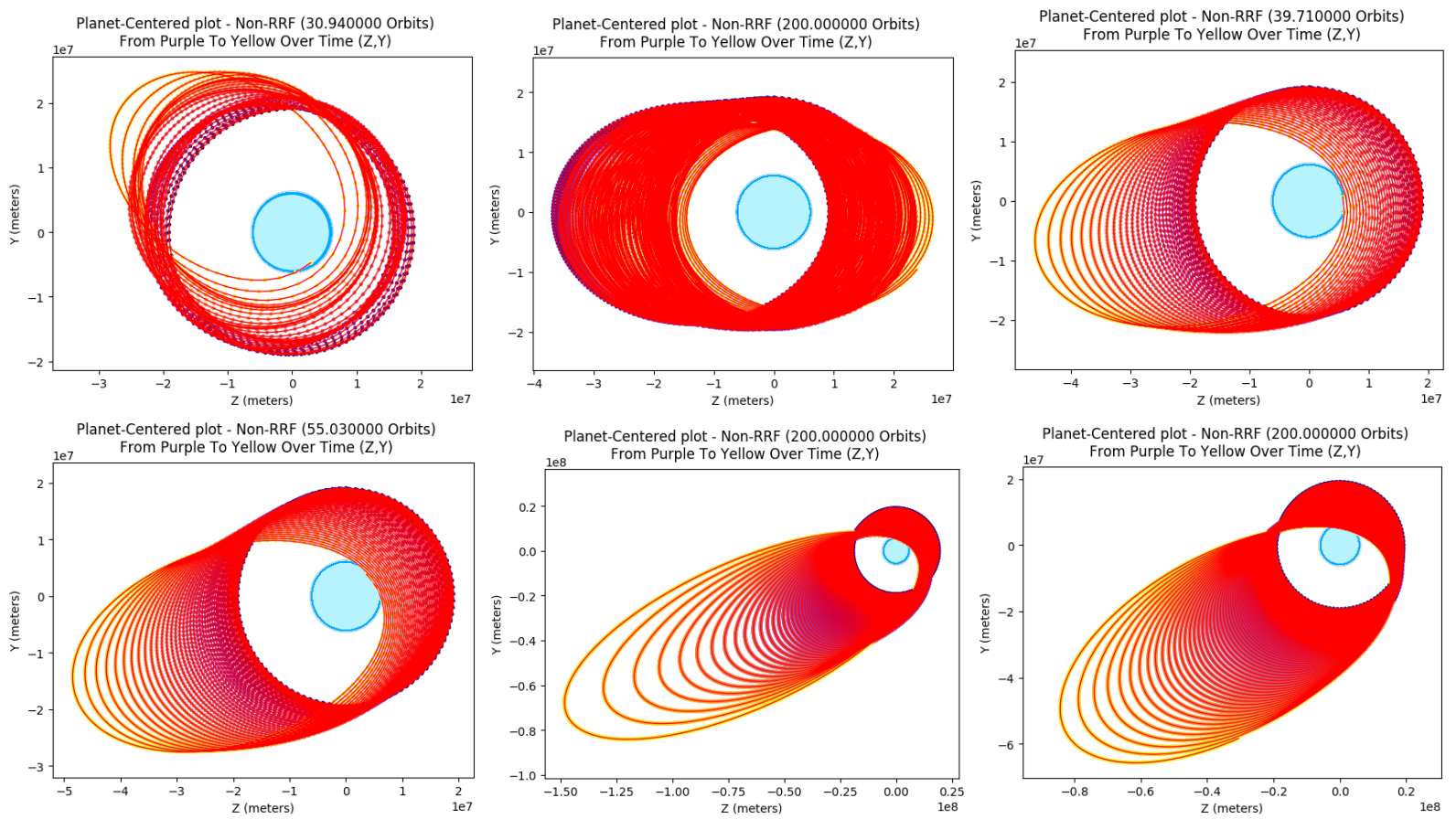

Fig. 6. These planet-centered plots in the ZY plane are for Night RP situations with the mirror 3 Earth radii away and in an initially face-on (ZY) orbit at the inner HZ. Stars increase in power from left to right. The mirror orbit elongates more quickly for more massive stars.

We found that Always RP sometimes outlasts Night RP, despite mirrors experiencing more time exposed to RP with Always RP. As an example, both panels of Fig. 7 are for a mirror 2 Earth radii away from the Earth-sized planet in an initially face-on orbit (ZY) around a G2 star. Each graph shows the mirror's distance from the planet during the simulation. The Always RP situation lasts the full 1000 mirror orbits requested while the Night RP situation lasts only 266.48 mirror orbits.
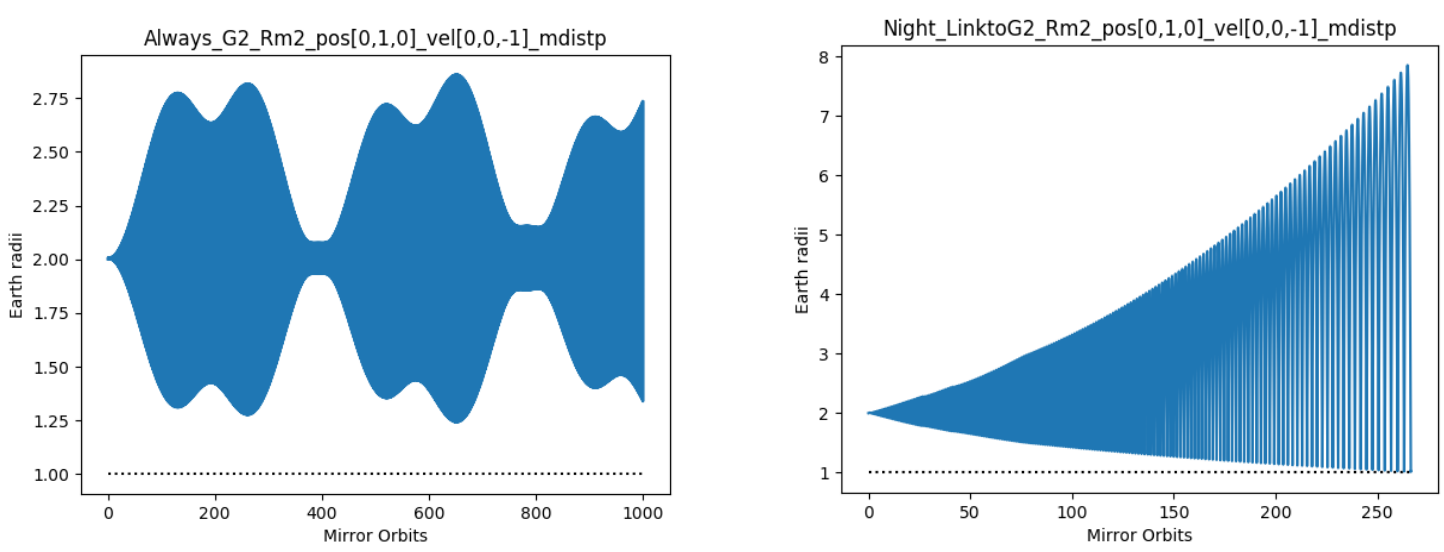
Fig. 7. The left situation (G2 star, $R_{m}=2$ Earth radii, mirror in $\mathrm{ZY}$ plane) has Always RP applied and the mirror survives the full 1000 orbits requested. Long-term, it appears relatively stable as the mirror distance to the planet is cyclical. The right situation has Night RP applied. The mirror is unstable and crashes into the planet after 266.48 orbits. The horizontal line indicates the radius of the planet. If the distance falls below that line, the mirror crashes.

Night RP situations did not always last longer than Always RP, because sometimes Always RP offsets its own RP effects at different points in the mirror orbit. This happens because RP tends to elongate the mirror orbit, but as the planet travels around its star, the direction of RP changes relative to the mirror orbit axis. Therefore, taken on their own, Always RP and Night RP have no systematic impact on mirror survival time.

There are no direct, monotonic trends other than the obvious. For example, mirrors where $\mathrm{R}_{\mathrm{m}}=10$ Earth radii are more unstable than for $\mathrm{R}_{\mathrm{m}}=2$ or 3 Earth radii, because the planet's gravitational pull is weaker. More statistical, multivariate analysis is needed to find trends between the initial parameters and the outcome.

To identify key parameters affecting the mirror orbit lifetime, we tabulated the mirror survival time in mirror orbits for each situation, along with initial orbital orientation, RP type, the number of mirror orbits per planet orbit $\left(P_{o r b, p l} / P_{o r b, \text { mirr }}\right)$, the ratio of RP acceleration to gravitational acceleration by the planet $\left(a_{R P} / a_{\text {Planet }}\right)$, and the equivalent ratio $\left(a_{R P} / a_{S t a r}\right)$ for the star. For these ratios, the RP acceleration assumed the mirror was face-on to the star. Multiple logistic regression was used to model the survival probabilities for various conditions. Initial statistical modeling efforts suggest that all of these parameters are significant in predicting mirror survival beyond 200 orbits. However not all relationships are monotonic, and some parameters are significant only in how they interact with others. We plan to re-analyze the results with 1000 mirror orbits as the maximum simulation time, and then extend the model to predict survival time based on input parameters.

\section{Conclusions}

We conclude that RP has a drastic impact on mirror stability because it adds an additional force to the system that is not offset by any other forces in the system

In the presence of RP, mirror orbits that were initially face-on to the star typically had the greatest survival time, because the RP effects were smaller and relatively consistent during the mirror orbit.

Mirror orbits for planets around M-type stars had a lot of precession, but this was not true for Gtype stars. This is because of the ratio of mirror orbits to planet orbits. M-type star planets travel around their star faster. For an M8, there are about 13 mirror orbits to a planet orbit. However, for a G2 star there are 128 mirror orbits in a planet orbit. That means, for an M5 star, RP direction is changing dramatically within a few mirror orbits.

Differences in day-side mirror orientation between Always RP and Night RP can cause dramatic changes to the mirror survival time, but not always in the same sense. 
Statistical analysis confirms that important factors in determining mirror survival time include: initial orbit orientation, the strength RP relative to the relevant gravitational forces, and the number of mirror orbits per planet orbit.

\section{Future Work}

In the future we will implement corrective thrusters on the mirror to "nudge" it at certain points in its orbit to offset of effects of RP to maintain a circular orbit. This is why we first need to find stable orbits as then the corrective thrusters use would be at a minimum, hence "fuel-efficient". Evaluating the thrusts required to maintain mirror orbits will help us estimate the fuel requirements for such a fleet of mirrors. This software will also be useful for several extension projects.

One way to apply thrust to a mirror is to alter its orientation - which would change the illumination of the planet as well as the orbital outcomes for the mirror. In future we can investigate the RP implications for various options of planet illumination.

Another possibility is to consider initially elliptical mirror orbits. Also, we may consider a mirror about non-synchronously rotating planets and/or those not in habitable zones as civilizations may be terraforming currently inhabitable planets.

Our flexible package will also handle mirror orbits further than 10 planet radii away for $\mathrm{K}$ and $\mathrm{M}$ stars, such as mirrors orbiting at the Lagrangian points (the "parking spots" of the universe), or cooler F and G stars. Finally, the package could include stellar wind forces if desired.

\section{Acknowledgements}

In addition to the WSGC, I would like to thank Wisconsin Alliance for Minority Participation (WiscAMP) for funding the initial phases of the project. Simulations in this paper made use of the REBOUND code which can be downloaded freely at http://github.com/hannorein/rebound.

\section{References}

Bennett, Jeffrey O., et al. The Cosmic Perspective. 8th ed., Pearson, 2017.

Gaidos, E. "Transit detection of a 'starshade' at the inner Lagrange point of an exoplanet." Monthly Notices of the Royal Astronomical Society, vol. 469, no. 4, 4 May 2017, pp. 4455-4464., doi:10.1093/mnras/stx1078.

(KSG) Korpela, E. J., Sallmen, S. M., \& Leystra Greene, D. (2015). Modeling Indications of Technology in Planetary Transit Light Curves-Dark-side Illumination. The Astrophysical Journal, 809(2), 139. doi:10.1088/0004-637X/ 809/2/139

Marcy, G. W., Isaacson, H., Howard, A. W., et al. 2014, ApJS, 210, 20 DOI:10.1088/0067$0049 / 210 / 2 / 20$

Petigura, E. A., Howard, A. W., and Marcy, G. W. 2013, Proceedings of the National Academy of Science, 110, 19273 ADS: 2013PNAS..11019273P 
Rein, H., and Liu, S. (2012). REBOUND: an open-source multi-purpose N- body code for collisional dynamics. Astronomy and Astrophysics, 537, A128. doi: 10.1051/0004$6361 / 201118085$

Rein, H., and Spiegel, D. S. (2014). Ias15: a fast, adaptive, high-order integrator for gravitational dynamics, accurate to machine precision over a billion orbits. Monthly Notices of the Royal Astronomical Society, 446(2), 1424-1437. doi:10.1093/mnras/stu2164

Weiss, L. M., and Marcy, G. W. (2014). The Mass-Radius Relation For 65 Exoplanets Smaller Than 4 Earth Radii. The Astrophysical Journal, 783(1), L6. doi:10.1088/2041$8205 / 783 / 1 /$ L6 